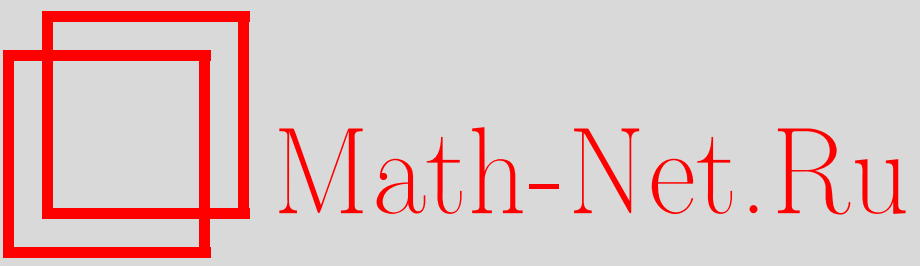

О. Ю. Марьясин, А. С. Колодкина, Иерархическое оптимальное управление со случайными ограничениями на ресурсы, Итоги науки и техн. Сер. Соврем. мат. и ее прил. Темат. обз., 2020, том 186, 91-101

DOI: https://doi.org/10.36535/0233-6723-2020-186-91-101

Использование Общероссийского математического портала Math-Net.Ru подразумевает, что вы прочитали и согласны с пользовательским соглашением

http: //www.mathnet.ru/rus/agreement

Параметры загрузки:

IP : 18.234 .197 .8

26 апреля 2023 г., 03:15:04 


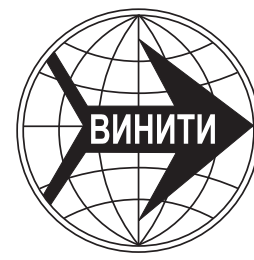

ИТОГИ НАУКИ И ТЕХНИКИ.

Современная математика и ее приложения.

Тематические обзоры.

Том 186 (2020). C. 91-101

DOI: 10.36535/0233-6723-2020-186-91-101

УДК 517.977 .5

\title{
ИЕРАРХИЧЕСКОЕ ОПТИМАЛЬНОЕ УПРАВЛЕНИЕ \\ СО СЛУЧАЙНЫМИ ОГРАНИЧЕНИЯМИ НА РЕСУРСЫ
}

\author{
(c) 2020 г. $\quad$ О. Ю. МАРЬЯСИН, А. С. КОЛОДКИНА
}

\begin{abstract}
Аннотация. Рассмотрен способ решения задачи оптимального управления сложной динамической системой со случайными ограничениями на ресурсы. Способ основан на использовании метода иерархической оптимизации, включающего итеративное решение локальных задач и задачи координации. Для решения локальных задач применяется подход, получивший название «управление с прогнозирующими моделями» (model predictive control). Учет случайных ограничений на ресурсы приводит к решению одноэтапных задач стохастического программирования.
\end{abstract}

Ключевые слова: сложная система, иерархическое оптимальное управление, стохастическое программирование.

\section{HIERARCHICAL OPTIMAL CONTROL WITH RANDOM RESOURCE CONSTRAINTS}

\author{
(c) 2020 O. YU. MARYASIN, A. S. KOLODKINA
}

\begin{abstract}
We consider a method for solving the problem of optimal control of a composite dynamic system with random resource constraints. The method is based on the method of hierarchical optimization, which includes iterative solution of local problems and the coordination problem. To solve local problems, we apply the model predictive control. Taking into account random resource constraints leads to the solution of one-stage problems of stochastic programming.
\end{abstract}

Keywords and phrases: complex system, hierarchical optimal control, stochastic programming.

AMS Subject Classification: 34H05, 49K15, 49N10, 93E20

1. Постановка задачи. Рассмотрим сложную динамическую систему, состоящую из $N$ взаимосвязанных однотипных элементов (подсистем). Под однотипностью здесь подразумевается то, что элементы имеют одинаковую природу, но могут различаться по своим параметрам. Выходы элементов являются входами для соседних элементов. Ситуация когда выход элемента непосредственно подается на его вход (рецикл), исключается. Динамика системы в дискретном времени описывается системой линейных уравнений с постоянными коэффициентами

$$
\begin{gathered}
x_{i k+1}=A_{i i} x_{i k}+B_{i} u_{i k}+\sum_{\substack{j=1, j \neq i \\
N}}^{N} A_{i j} x_{j k}+G_{i} w_{i k}, \\
y_{i k}=C_{i} x_{i k}
\end{gathered}
$$

где $x_{i k} \in \mathbb{R}^{n_{x i}}$ - вектор переменных состояния $i$-го элемента $i=1, \ldots, N$, в $k$-й момент времени $k=1, \ldots, N_{t}, N_{t}=T / h, T$ - интервал, на котором осуществляется управление, $h$ - шаг дискретизации, $u_{i k} \in \mathbb{R}^{n_{u i}}$ - вектор управлений $i$-го элемента, $y_{i k} \in \mathbb{R}^{n_{y i}}$ - вектор выходных измеряемых переменных $i$-го элемента, $w_{i k}$ - вектор ограниченных, контролируемых возмущений, действующих на $i$-й элемент. 
Задача оптимального управления системой (1) ставится в виде

$$
J=\sum_{i=1}^{N} J_{i} \rightarrow \min _{u}
$$

с учетом ограничения (1) и дополнительных ограничений

$$
\begin{gathered}
y_{i k \min } \leqslant y_{i k} \leqslant y_{i k \max }, \quad i=1, \ldots, N, \quad k=1, \ldots, N_{t}, \\
0 \leqslant r_{i k}\left(u_{i k}\right) \leqslant r_{i k \max }, \quad i=1, \ldots, N, \quad k=0, \ldots, N_{t}-1, \\
\sum_{i=1}^{N} r_{i m}\left(u_{i m}\right) \leqslant U_{m k}, \quad m=1, \ldots, M,
\end{gathered}
$$

где вектор $r_{i}\left(u_{i}\right)$ определяет количество ресурсов, выделяемых $i$-му элементу, $r_{i}\left(u_{i}\right)=$ $\left\{r_{i 1}\left(u_{i 1}\right), r_{i 2}\left(u_{i 2}\right), \ldots, r_{i m}\left(u_{i m}\right), \ldots, r_{i M}\left(u_{i M}\right)\right\}$, где $r_{i m}\left(u_{i m}\right)$ - расход $m$-го вида ресурса для $i$-го элемента, $U_{m k}$ - общее количество $m$-го вида ресурса, выделяемого системе, $M$ - число видов ресурсов. Зависимость $r_{i}\left(u_{i}\right)$ принимается линейной, вида $r_{i}=d_{i} u_{i}$, где $d_{i}$-масштабный коэффициент. Ограничение (3) задает пределы изменения контролируемых параметров $i$-го элемента. Ограничение (4) определяет максимальное количество ресурса для $i$-го элемента. Глобальное ограничение (5) определяет общее количество $m$-го вида ресурса для всех элементов.

Критерий $J_{i}$ имеет вид

$$
J_{i}=\sum_{k=1}^{N_{t}}\left[y_{i k}-y_{i k z}\right]^{\top} Q_{i}\left[y_{i k}-y_{i k z}\right]+\sum_{k=1}^{N_{t}-1} u_{i k}^{\top} R_{i} u_{i k},
$$

где $y_{i k z}$ - вектор заданных значений параметров $i$-го элемента, $Q_{i}$ - симметрическая неотрицательно определенная матрица, $R_{i}$ - диагональная положительно определенная матрица.

2. Основные результаты. При высокой размерности задачи (2) с ограничениями (1), (3)-(5) ее решение может быть связано с большими вычислительными затратами. Для решения сложных задач оптимизации с аддитивно-сепарабельным видом критерия оптимальности и с блочнодиагональной структурой части ограничений традиционно используются методы иерархической оптимизации (декомпозиционные методы оптимизации). Эти методы в основном были разработаны в 60-80-х годах и детально описаны в $[1-4,7]$. В литературе нет общепринятых названий для популярных методов иерархической оптимизации. Так, один и тот же метод в [1] назван методом явной декомпозиции, в [3]- методом прогнозирования взаимодействий, в [4] - методом координации моделей, в [7] - прямым методом. Далее мы будем придерживаться терминологии, принятой в [1]. Одним из существенных преимуществ метода явной декомпозиции перед другими методами, например, методом неявной декомпозиции (называемым еще методом согласования взаимодействий [3], методом целевой координации [4] или методом баланса взаимодействий [7]), является то, что значения управлений, полученные в ходе решения задачи иерархической оптимизации можно сразу использовать для управления процессом в «реальном времени», так как при этом удовлетворяются все ограничения задачи. Поэтому для решения поставленной задачи мы будем использовать метод явной декомпозиции.

В соответствии с методом явной декомпозиции решение глобальной задачи (2) с ограничениями (1), (3)-(5) разбивается на решение $N$ локальных задач

$$
J_{i} \rightarrow \min _{u_{i}}, \quad i=1, \ldots, N,
$$

с ограничениями с $(1),(3),(4)$. Если принять, что $A_{i j}=L_{j} C_{j}$, то уравнение состояния в $(1)$ может быть представлено в виде

$$
x_{i k+1}=A_{i i} x_{i k}+B_{i} u_{i k}+\sum_{\substack{j=1 \\ j \neq i}}^{N} L_{j} y_{j k}+G_{i} w_{i k} .
$$


Зафиксировав значения входов в подсистемы на уровне $y_{j k}^{*}$ получаем систему из $N$ независимых подсистем.

Задачи координации в этом случае будет включать критерий

$$
J=\sum_{i=1}^{N} J_{i} \rightarrow \min _{c}
$$

где $c=\left\{y_{i k}, r_{i k \max }\right\}$ - вектор координирующих переменных, и глобальные ограничения (5).

Для решения локальных задач (7) с ограничениями (1), (3), (4) будем использовать подход, получивший название Model Predictive Control (MPC) [13]. K достоинствам MPC-подхода можно отнести то, что оптимальный регулятор, синтезированный в соответствии с данным подходом, обеспечивает отсутствие в системе статической ошибки, соблюдение ограничений на управляющие и выходные переменные и обеспечивает компромисс между робастностью и качеством регулирования. Совместное использование методов иерархической оптимизации и МРС-подхода для управления сложными, распределенными системами привело к появлению иерархических распределенных МРС-алгоритмов [12].

Известно [13], что решение задачи оптимального управления с квадратичным критерием типа (6) и ограничениями (1), (3), (4) с использованием МРС-подхода, сводится к решению задачи квадратичного программирования

$$
\begin{aligned}
& J_{i}=v_{i}^{\top} H_{i} v_{i}+f_{i}^{\top} v_{i} \rightarrow \min _{v_{i}}, \\
& L_{y i} v_{i} \leqslant b_{y i}, \quad i=1, \ldots, N, \\
& L_{u i} v_{i} \leqslant b_{u i}, \quad i=1, \ldots, N,
\end{aligned}
$$

где

$$
v_{i}=\left(\begin{array}{c}
u_{i 0} \\
u_{i 1} \\
\vdots \\
u_{i N_{l}-1}
\end{array}\right),
$$

$N_{l}$ - число шагов дискретизации на горизонте прогнозирования локальных задач $T_{l}$, а матрицы $H_{i}, f_{i}, L_{y i}, b_{y i} L_{u i}, b_{u i}$ зависят от матриц модели $A_{i}, A_{l}, B_{i}, G_{i}, C_{i}$, весовых матриц $Q_{i}, R_{i}$ и векторов ограничений $y_{i k \min }, y_{i k \max }, r_{i k \max }$. Отметим, что МРС-алгоритмом на текущем шаге реализуется только управление $u_{i 0}$, после чего горизонт прогнозирования сдвигается на следующий такт времени, он становится текущим и процесс повторяется.

Если при фиксированных значениях координирующих воздействий существуют решения локальных задач (9), то условие устойчивости рассмотренной системы иерархического распределенного управления на базе МРС-подхода совпадает с условием устойчивости, полученным для распределенных МРС-алгоритмов [6]. В этом случае уравнение состояния в (1) представляется в виде

$$
x_{i k+1}=A_{i i} x_{i k}+B_{i} u_{i k}+\sum_{\substack{j=1 \\ j \neq i}}^{N} A_{i j} \tilde{x}_{j k}+G_{i} w_{i k}
$$

где $\tilde{x}_{j k}$ - предсказанные значения вектора состояния для $j$-х соседей $i$-го элемента. Основным предположением, используемым при выводе условий устойчивости в [6] является предположение о том, что найдутся такие матрицы $K_{i} \in \mathbb{R}^{n_{x i} \times n_{y i}}$, что при отсутствии внешних воздействий матрицы $A_{i}+B_{i} K_{i}$ являются устойчивыми по Шуру, и матрица $A+B K$, где матрицы $A$ и $B$ составлены из элементов $A_{i j}$ и $B_{i}$, а $K=\operatorname{diag}\left(K_{1}, K_{2}, \ldots, K_{N}\right)$, также является устойчивой по Шуру.

Структурная схема иерархической распределенной системы управления (ИРСУ) показана на рис. 1. Локальные системы управления (ЛСУ) решают локальные задачи (9) и реализуют управление своими подсистемами. Матрица $L$ используется для описания взаимосвязей между подсистемами. Элемент матрицы $l_{i j}$ равен единице, если выход подсистемы $i$ поступает на вход 


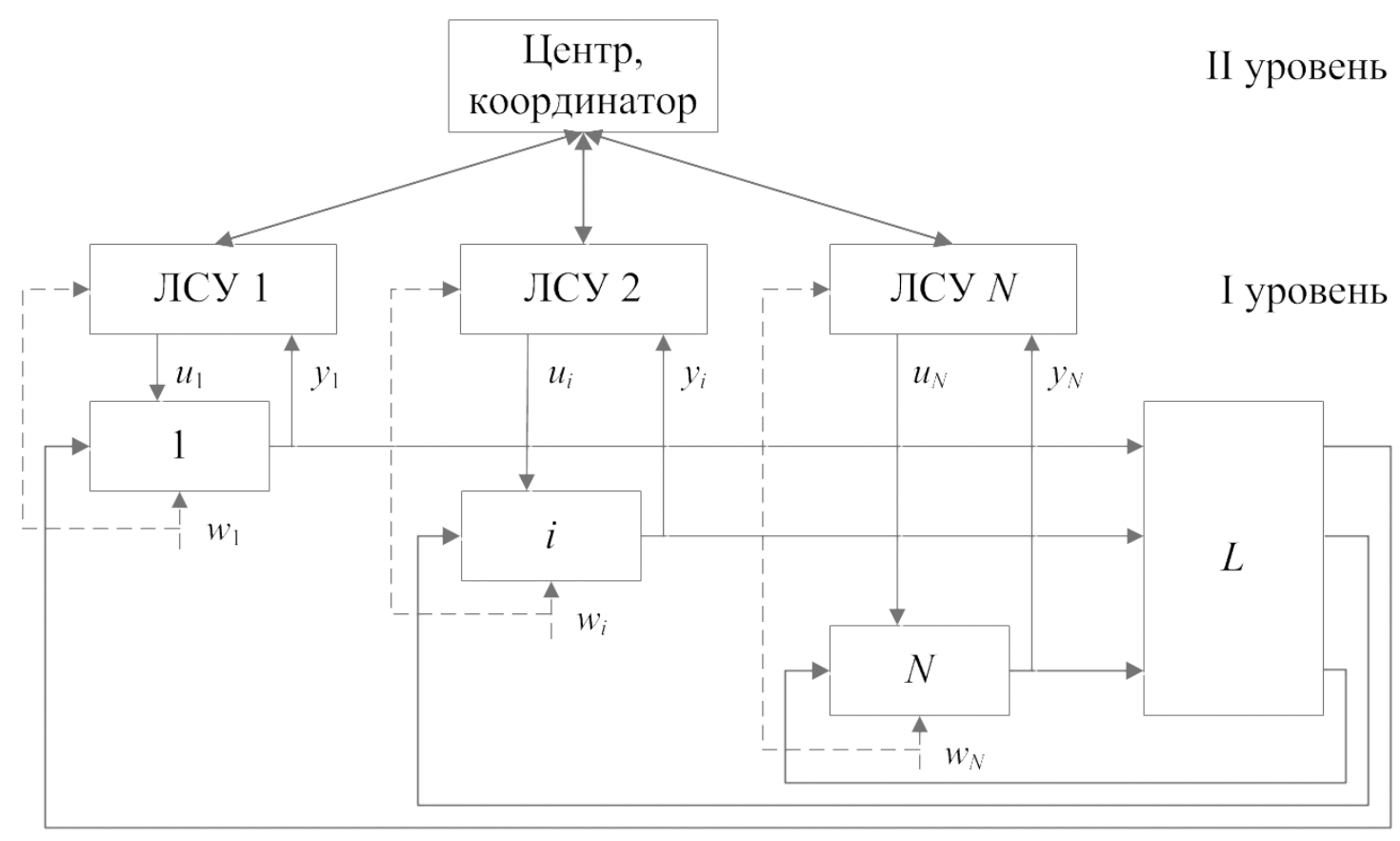

Рис. 1. Структура иерархической системы управления

подсистемы $j$ и равен нулю, если связь отсутствует. Кроме того, как было сказано ранее, связанными могут быть только соседние подсистемы и отсутствуют связи с выходов подсистем на их же входы.

Центр (координатор) решает задачу координации (8) с учетом глобальных ограничений (5).

Рассмотрим временные последовательности решения задач и реализации управления в ИРСУ. Очевидно, что период решения задачи координации $T_{p}$ должен быть больше периода решения локальных задач $T_{l}$. Предположим, что изменения внешних условий, приводящие к необходимости решения задачи оптимального управления (синтеза оптимального регулятора) представляют собой достаточно инерционные процессы с периодом, большим $T_{p}$. Обозначим $T_{s}$ время решения задачи координации. Желательно чтобы это время не превышало $T_{p}$. Разобьем интервал $T$ на $P$ подинтервалов, так, чтобы в каждый подинтервал укладывалось целое число шагов дискретизации $N_{p}$ и выполнялось равенство $N_{p} P=N_{t}$. Решение задачи координации будем производить в моменты $l=0, N_{p}, 2 N_{p}, \ldots,(P-1) N_{p}$, а решения локальных задач $(7)$ с ограничениями $(1),(3)$, (4) в моменты времени $k=l, N_{l}, \ldots, N_{p}$.

В момент времени $T_{s}$ заканчивается синтез глобально-оптимального управления, которое будет действовать на интервале $T_{p}-T_{s}$. Следовательно, в течение времени $T_{s}$ управление не будет глобально оптимальным, хотя и будет удовлетворять ограничениям (5). Как уже было сказано ранее, это выгодно отличает метод явной декомпозиции от других методов, для которых реализация управления возможна только после решения задачи координации. Таким образом, для повышения качества управления необходимо добиваться уменьшения времени решения задачи координации.

Сложность решения задачи координации сильно зависит от ее размерности. Поэтому желательно сократить число координирующих переменных. Требование приближения значений переменных $y_{i k}$ к заданным значениям $y_{i k z}$, выраженное в критерии (6), побуждает к заданию связей между подсистемами, равными $y_{i k z}$. Таким образом, вектор координирующих переменных будет включать только вектор $r_{i k \max }$.

Для решения локальных задач (9) от верхнего уровня (задачи координации) требуется только часть элементов $b_{u i z}$ вектора $b_{u i}$, относящихся к вектору $r_{i k \max }$. В результате решения локальных 
задач получаем значения оптимального управления $v_{i m}^{*}$, связанного с расходом $m$-го вида ресурсов для $i$-й подсистемы при ограничениях $b_{u i m}, m=1, \ldots, M$, и значение критерия оптимальности $i$-й зоны $J_{i}\left(b_{u i z}\right)$. Координатор использует информацию о $J_{i}\left(b_{u i z}\right)$ от подсистем нижнего уровня для решения задачи координации в виде

$$
\begin{gathered}
J=\sum_{i=1}^{N} J_{i}\left(b_{u i z}\right) \rightarrow \min _{b_{u i z}} \\
\sum_{i=1}^{N} b_{u i m} \leqslant U_{m}, \quad m=1, \ldots, M .
\end{gathered}
$$

Введем множества

$$
D_{i}=\left\{v_{i} \mid L_{y i} v_{i} \leqslant b_{y i}\right\}, \quad V_{i}=\left\{b_{u i} \mid \exists v_{i} \in D_{i}: L_{u i} v_{i} \leqslant b_{u i}\right\} .
$$

Теорема 1. Пусть выполнены следующие условия:

(1) множества $D_{i}$ непусты, компактны и выпуклъ;

(2) бункици $J_{i}\left(b_{\text {иіz }}\right)$ и $L_{u i} v_{i}$ непрерьвнь и выпукль на $D_{i}$;

(3) задача (9) имеет допустимое решение.

Тогда задача координации (10), (11) имеет допустимое оптимальное решение.

Доказательство. Предположения о выпуклости $J_{i}\left(b_{u i z}\right), L_{u i} v_{i}$ и $D_{i}$ обеспечивают, что множество $V_{i}$ выпукло и что $J_{i}\left(b_{u i z}\right)$ выпукла на $V_{i}$, так что задача координации является задачей выпук-

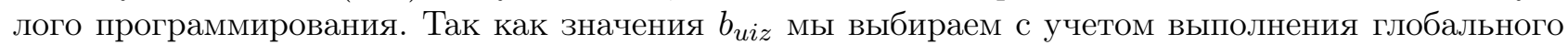
ограничения, то допустимость решения задачи (9) приводит к тому, что координирующая задача также имеет допустимое решение. Из компактности $D_{i}$ вместе с непрерывностью $J_{i}\left(b_{u i z}\right)$ и $L_{u i} v_{i}$ следует, что $i$-я подзадача имеет оптимальное решение, поскольку она имеет допустимое. Следовательно, координирующая задача также будет иметь оптимальное решение.

Для решения локальных задач (9) могут быть использованы эффективные методы решения задач квадратичного программирования. Функции $J_{i}\left(b_{u i z}\right)$ могут быть недифференцируемы в некоторых точках по переменным $b_{\text {uiz }}$, и разрывы производных могут оказаться в критических точках, например, в минимуме. Поэтому для решения координирующей задачи (10), (11) могут использоваться методы недифференцируемой оптимизации, например, метод возможных направлений или Бандл-метод [8].

Предположим, что общее количество $m$-го вида ресурса $U_{m}$ является случайной величиной с известным законом распределения. Тогда глобальное ограничение в задаче координации может быть записано как

$$
P\left(\sum_{i=1}^{N} b_{u i m} \leqslant U_{m}\right) \geqslant \alpha_{m}, \quad m=1, \ldots, M,
$$

где $\alpha_{m}$ - заданная вероятность соблюдения ограничения, $0 \leqslant \alpha_{m} \leqslant 1$. Задачу (10), (12) можно будет трактовать как задачу стохастического программирования. Для решения данной задачи будем использовать одноэтапную стратегию [5]. Если случайные величины $U_{m}$ характеризуются функцией распределения $F_{m}\left(U_{m}\right)$, то значение $\tilde{U}_{m}$ представляет собой наибольшее число удовлетворяющее неравенству $1-F_{m}\left(\tilde{U}_{m}\right) \geqslant \alpha_{m}$. Если $F_{m}\left(U_{m}\right)$ непрерывная, строго монотонная функция, то последнее неравенство эквивалентно уравнению $1-F_{m}\left(\tilde{U}_{m}\right)=\alpha_{m}$. Следовательно, $\tilde{U}_{m}$ может быть определена как $\tilde{U}_{m}=F^{-1}\left(1-\alpha_{m}\right)$. Кроме того, $\tilde{U}_{m}$ можно найти из решения уравнения

$$
\int_{\tilde{U}_{m}}^{\propto} \phi_{m}\left(U_{m}\right) d U_{m}=\alpha_{m}, \quad m=1, \ldots, M
$$


где $\phi_{m}\left(U_{m}\right)$ - плотность распределения компоненты $U_{m}$. Тогда задача координации будет состоять в минимизации критерия (10) с ограничением

$$
\sum_{i=1}^{N} b_{\text {uim }} \leqslant \tilde{U}_{m}, \quad m=1, \ldots, M .
$$

Теперь предположим, что вместо ограничения (4) имеется ограничение

$$
r_{i m k}\left(u_{i m k}\right)+\tilde{r}_{i m} \leqslant r_{i m \max }, \quad i=1, \ldots, N, \quad m=1, \ldots, M, \quad k=0, \ldots, N_{l}-1,
$$

где $\tilde{r}_{i m k}-$ случайная величина с известным законом распределения. Последнее неравенство перепишем в виде

$$
r_{i m k}\left(u_{i m k}\right) \leqslant r_{i m \max }-\tilde{r}_{i m}, \quad i=1, \ldots, N, \quad m=1, \ldots, M, \quad k=0, \ldots, N_{l}-1 .
$$

Тогда аналогично выражению (12) можно записать

$$
P\left(r_{i m k}\left(u_{i m k}\right) \leqslant \tilde{r}_{i m \max }\right) \geqslant \beta_{i m}, \quad i=1, \ldots, N, \quad m=1, \ldots, M, \quad k=0, \ldots, N_{l}-1,
$$

где $\beta_{i m}$ - заданные вероятности соблюдения ограничений, $0 \leqslant \beta_{i m} \leqslant 1$. Рассматривая задачу с вероятностными ограничениями (14) как задачу стохастического программирования перепишем задачу квадратичного программирования (10) в виде

$$
\begin{gathered}
J_{i}=v_{i}^{\top} H_{i} v_{i}+f_{i}^{\top} v_{i} \rightarrow \min _{v_{i}}, \\
L_{y i} v_{i} \leqslant b_{y i}, \quad i=1, \ldots, N, \\
\hat{L}_{u i} v_{i} \leqslant \hat{b}_{u i}, \quad i=1, \ldots, N, \\
\tilde{L}_{u i} v_{i} \leqslant \tilde{b}_{u i}, \quad i=1, \ldots, N,
\end{gathered}
$$

где матрицы $\hat{L}_{u i}, \tilde{L}_{u i}$ и векторы $\hat{b}_{u i}, \tilde{b}_{u i}$ получаются из элементов матрицы $L_{u i}$, векторов $b_{u i}$ и $\tilde{r}_{\text {immax }}$ соответственно.

Аналогично детерминированному МРС-алгоритму к задаче квадратичного программирования типа (15) приводит стохастический МРС-алгоритм с вероятностными ограничениями [9]. Таким образом, исходная задача оптимального управления (1)-(5) при случайных ограничениях на ресурсы сводится к интерактивному решению задачи координации $(10),(13)$ и локальных задач (15).

3. Пример. Рассмотрим задачу управления микроклиматом и энергопотреблением здания, состоящего из множества зон (секций, помещений). Так как зоны здания имеют общие ограждающие конструкции, то на температуру в каждой зоне оказывает влияние температура в соседней зоне. Это влияние тем сильнее, чем выше разность температур в соседних зонах, больше площадь общих ограждающих конструкций и выше их коэффициенты теплопередачи. Для простоты будем полагать, что зоны имеют одинаковый материал внешних стен и перекрытий между зонами.

Динамика микроклимата здания без учета термической массы ограждающих конструкций (стен, окон, пола, потолка), инфильтрации тепла, влаги и газов через ограждающие конструкции, солнечной радиации и бытовых тепло-, влаго- и газовыделений может быть описана системой дифференциальных уравнений

$$
\begin{gathered}
c \rho V_{i} \frac{d T_{i}}{d t}=q_{i}+c G_{n i}\left(T_{n i}-T_{i}\right)+\sum_{\substack{s=1 \\
s \neq i}}^{S_{i}} k_{i n} F_{s}^{i n}\left(T_{s}-T_{i}\right)+\sum_{r=1}^{R_{i}} k_{e} F_{r}^{e x t}\left(T_{E}-T_{i}\right), \quad i=1, \ldots, N, \\
\rho V_{i} \frac{d W_{i}(t)}{d t}=G_{n i}\left(W_{n i}(t)-W_{i}(t)\right), \quad i=1, \ldots, N, \\
\rho V_{i} \frac{C_{i}(t)}{d t}=G_{n i}\left(C_{n i}(t)-C_{i}(t)\right), \quad i=1, \ldots, N,
\end{gathered}
$$

где $c$ - теплоемкость воздуха; $\rho$ - плотность воздуха; $V_{i}$ - объем $i$-й зоны, $i=1,2, \ldots, N ; T_{i}-$ температура $i$-й зоны, ${ }^{\circ} \mathrm{C} ; t$ - время; $q_{i}$ - тепло, полученное от системы теплоснабжения $i$-й зоны; $G_{n i}$ - расход приточного воздуха $i$-й зоны; $T_{n i}$ - температура приточного воздуха $i$-й зоны; $S_{i}$ - число внутренних ограждающих конструкций $i$-й зоны; $k_{i n}$ - коэффициент теплопередачи 


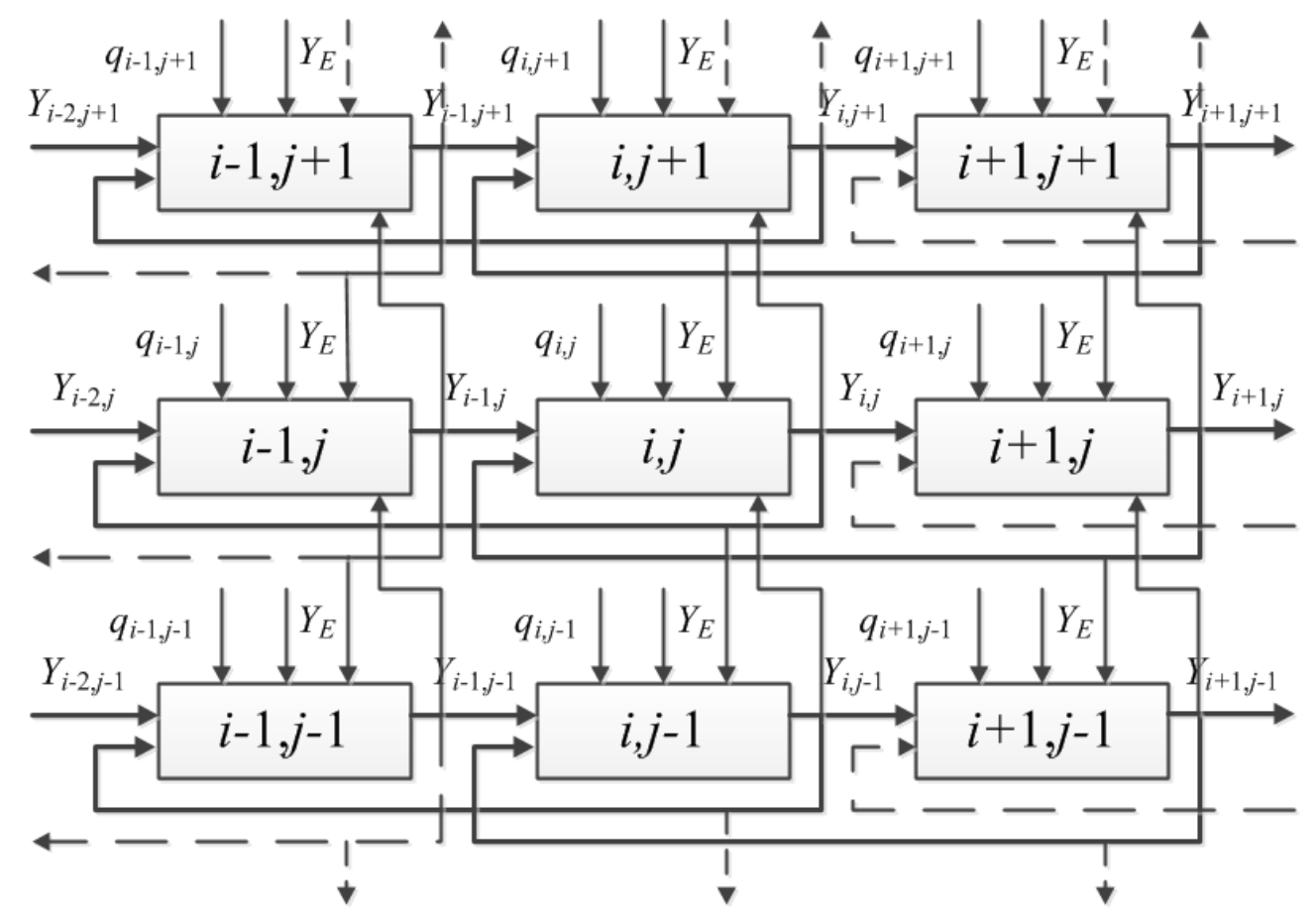

Рис. 2. Структура модели здания

ограждающих конструкций между зонами; $F_{s}^{i n}$ - площадь ограждающих конструкций между зонами, $s=1,2, \ldots, S_{i} ; R_{i}$ - число внешних ограждающих конструкций $i$-й зоны; $k_{e}$ - коэффициент теплопередачи внешних ограждающих конструкций $i$-й зоны; $F_{r}^{e x t}$ - площадь внешних ограждающих конструкций $i$-й зоны, $r=1,2, \ldots, R_{i} ; T_{E}$ - температура наружной среды; $W_{i}$ - влажность воздуха $i$-й, г; $W_{n i}$-влажность приточного воздуха $i$-й зоны; $C_{i}$ - концентрация $\mathrm{CO}_{2}$ в воздухе $i$-й зоны, ppm; $C_{n i}$ - концентрация $\mathrm{CO}_{2}$ в приточном воздухе $i$-й зоны.

Структура модели микроклимата здания, соответствующая системе (16), для двухмерного случая описывается схемой, фрагмент которой показан на рис. 2. Здесь индекс $i$ для удобства восприятия разделен на два индекса: индекс $i$ действует по горизонтали, $j$ - по вертикали. Вектор $Y_{i j}=\left\{T_{i j}, W_{i j}, C_{i j}\right\}$ - вектор параметров микроклимата здания. Вектор $Y_{E}=\left\{T_{E}, W_{E}, C_{E}\right\}-$ вектор параметров наружной среды. Такая структура соответствует, например, многоэтажному зданию с последовательным расположением зон. В общем случае структура модели будет описываться трехмерной схемой.

Задача оптимального управления энергопотреблением и микроклиматом здания после дискретизации может быть записана в виде

$$
J=\sum_{i=1}^{N} J_{i} \rightarrow \min _{q}
$$

где

$$
J_{i}=\sum_{k=1}^{N_{t}}\left[Y_{i}-Y_{i z}\right]^{\top} Q_{i}\left[Y_{i}-Y_{i z}\right]+\sum_{k=1}^{N_{t}-1} q_{i}^{\top} R_{i} q_{i},
$$

$Y_{i}=\left\{T_{i}, W_{i}, C_{i}\right\}$ - вектор параметров микроклимата здания в $i$-ой зоне, $Y_{i z}=\left\{T_{i z}, W_{i z}, C_{i z}\right\}-$ вектор заданных параметров микроклимата здания в $i$-ой зоне, $q_{i}=\left\{q_{i 1}, \ldots, q_{i m}, \ldots, q_{i M}\right\}-$ pacход энергоресурсов для $i$-й зоны; $q_{i m}$ - расход $m$-го вида энергоресурсов $i$-й зоны; $M$ - число видов энергоресурсов. 
В качестве ограничений выступают дискретно-разностные аналоги уравнения (16), локальные ограничения

$$
\begin{gathered}
T_{i \min } \leqslant T_{i} \leqslant T_{i \max }, \quad i=1, \ldots, N \\
\varphi_{i \min } \leqslant \varphi_{i} \leqslant \varphi_{i \max }, \quad i=1, \ldots, N, \\
C_{i} \leqslant C_{i \max }, \quad i=1, \ldots, N, \\
0 \leqslant q_{i m} \leqslant q_{i m \max }, \quad i=1, \ldots, N, \quad m=1, \ldots, M,
\end{gathered}
$$

и глобальное ограничение

$$
\sum_{i=1}^{N} q_{i m} \leqslant Q_{m}, \quad m=1, \ldots, M,
$$

где $T_{i \min }, T_{i \max }$ - минимальное и максимальное значение температур в помещении $i$-й зоны; $\varphi_{i \min }, \varphi_{i \max }-$ минимальное и максимальное значение относительной влажности в помещении $i$-й зоны; $C_{i \max }$ - максимальное содержание $\mathrm{CO}_{2}$ в воздухе $i$-й зоны; $q_{i m \max }-$ максимальное количество $m$-го вида энергоресурса на энергоснабжение $i$-й зоны; $\tilde{Q}_{m}-$ максимальное количество $m$-го вида энергоресурса на энергоснабжение всего здания.

Случайный характер ограничений (20), (21) происходит из-за расходования энергоресурсов на цели, не связанные с управлением микроклиматом. Поясним это для двух видов энергоресурсов на примере расходования тепловой и электрической энергии. В этом случае, учитывая то, что тепловая энергия может тратиться на горячее водоснабжение, а электрическая энергия используется для освещения и других бытовых нужд, можно записать

$$
\begin{gathered}
q_{i o}+q_{i g} \leqslant q_{i h \max }, \quad i=1, \ldots, N, \quad q_{i e}+q_{i b} \leqslant q_{i e \max }, \quad i=1, \ldots, N, \\
\sum_{i=1}^{N}\left(q_{i o}+q_{i g}\right) \leqslant q_{h \max }, \quad \sum_{i=1}^{N}\left(q_{i e}+q_{i b}\right) \leqslant q_{e \max },
\end{gathered}
$$

где $q_{i o}$ - расход тепла, используемый на отопление $i$-й зоны, $q_{i g}$ - расход тепла, используемый на горячее водоснабжение $i$-й зоны, $q_{i e}$ - расход электроэнергии, используемый на отопление $i$-й зоны, $q_{i b}$ - расход электроэнергии, используемый на другие бытовые нужды $i$-й зоны, $q_{i h \max }-$ максимальный расход тепла для $i$-й зоны, $q_{i e \max }$ - максимальный расход электроэнергии для $i$-й зоны, $q_{h \max }$ - максимальный расход тепла для всего здания, $q_{e} \max$ - максимальный расход электроэнергии для всего здания. Расходы $q_{i g}$ и $q_{i b}$ являются случайными и в часы пик могут достигать значительных значений.

С учетом случайного характера значений $q_{i m \max }$ и $Q_{m}$ ограничения $(20)$ и $(21)$ будут записаны как

$$
\begin{gathered}
q_{i m} \leqslant \tilde{q}_{i m \max }, \quad i=1, \ldots, N, \quad m=1, \ldots, M, \\
\sum_{i=1}^{N} q_{i m} \leqslant \tilde{Q}_{m}, \quad m=1, \ldots, M .
\end{gathered}
$$

Оценить плотности распределения вероятностей потребления энергоресурсов можно экспериментально, по показаниям соответствующих счетчиков. При этом необходимо учитывать, что потребление энергоресурсов может существенно отличатся для разных сезонов года и разных часов суток. Вопросы выбора подходящего закона распределения вероятностей и оценки его параметров выходят за рамки данной статьи. Пример оценки плотности распределения вероятностей потребления электроэнергии на основе гистограммы показан на рис. 3.

В качестве источников тепла рассматривалось центральное водяное отопления и электрическое отопление с возможностью местного регулирования температуры. Значение $T_{i \text { min }}$ принималось $20^{\circ} \mathrm{C}, T_{i \max }-22^{\circ} \mathrm{C}$. Для водяного отопления значение $q_{i m \max }$ принималось 20 кВт, для электрического отопления -5 кВт. Цены на энергоносители принимались по текущим тарифам на отопление и электроэнергию. Возмущения по параметрам внешней среды моделировались периодическими функциями на интервале времени от 0 до 24 ч. 


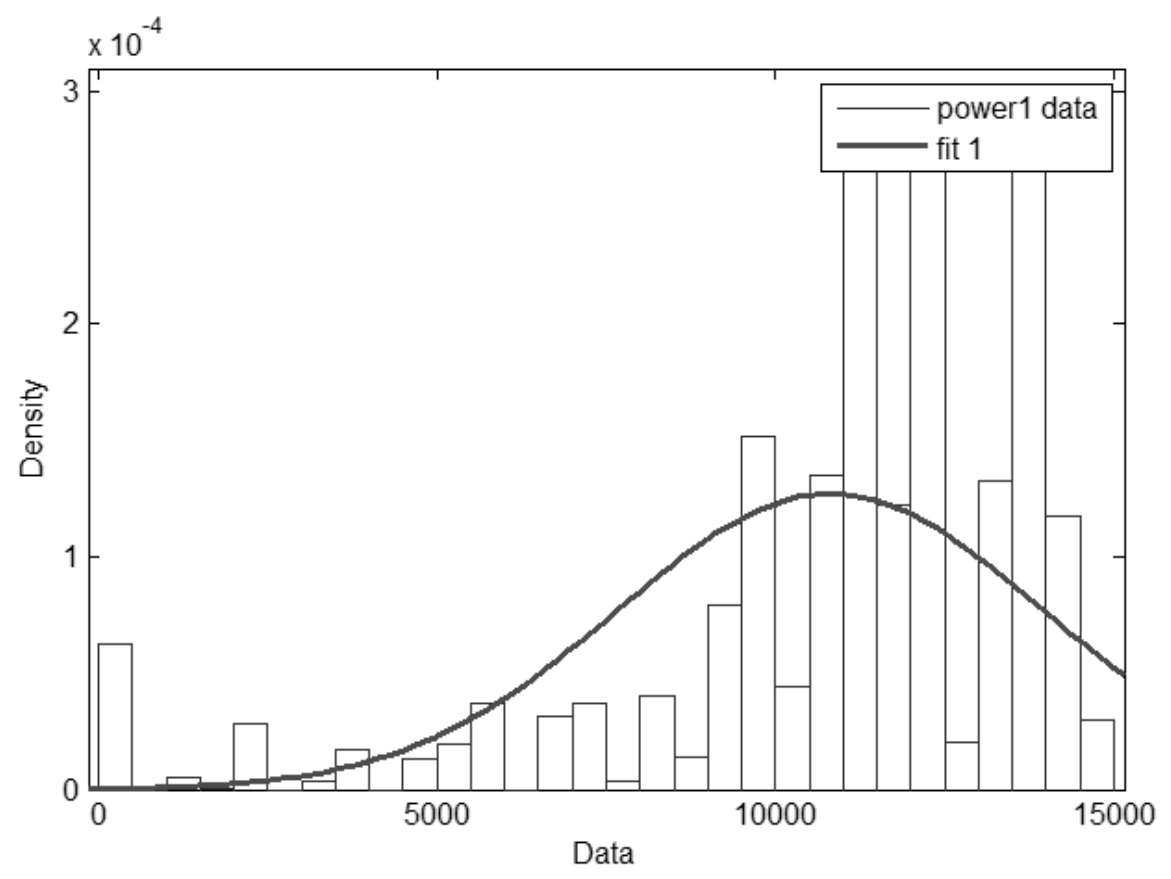

Рис. 3. Пример оценки плотности вероятности потребления электроэнергии

Для решения задачи (17) с ограничениями в виде дискретно-разностного аналога уравнения (16), и ограничениями (19), (22), (23) использовался изложенный в разделе 2 способ иерархического оптимального управления при случайных ограничениях на ресурсы. На рис. 4 показаны графики изменения, усредненных за час, расходов тепла от водяного и электрического отопления, в одной из зон, при иерархическом оптимальном управлении с тремя подсистемами при различных значениях расхода тепла на горячее водоснабжение $q_{i g}$. Сплошной линией показан расход тепла от водяного отопления, пунктирной - расход от электрического отопления.

Так как тариф на электроэнергию больше тарифа на отопление, то для поддержания температуры в основном расходуется тепловая энергия. Этой ситуации соответствует график, показанный на рис. 4(a). На рис. 4(b) показана ситуация когда из-за необходимости учета глобального ограничения (23) в часы пик на короткое время становится невозможным использование одного вида энергоресурсов. Для поддержания требуемого теплового режима в такие моменты резко возрастает расход альтернативного энергоносителя.

В литературе можно найти описание применения методов иерархической оптимизации для решения задач оптимального управления энергопотреблением и микроклиматом зданий с использованием МРС-подхода. В работе [10] описывается применение классического метода Данцига-Вульфа для решения задачи оптимального управления тепловым режимом многозонного здания. При этом задача, решаемая МРС-алгоритмом на каждом шаге, приводится к задаче линейного программирования. В работе [11] для решения задачи, подобной задаче в [10], используется метод декомпозиции Бендерса. Здесь так же, как и в [10], решается задача линейного программирования. В работе [8] для решения задачи оптимального управления микроклиматом здания используется метод декомпозиции путем распределения ресурсов, ранее описанный в [2]. При этом локальные задачи и задача координации представляют собой задачи линейного программирования. Линейность задач в работах $[8,10,11]$ обусловлена использованием линейного критерия оптимальности, отвечающего целям энергосбережения. В отличие от этого, квадратичный критерий (18) позволяет не только экономить энергоресурсы, но и обеспечивать требуемое качество параметров микроклимата в динамических режимах, например, при переходе с одного режима на другой, более энергосберегающий, при отсутствии людей в помещении в нерабочее время. Задачи решаемые в работах $[8,10,11]$ являются детерминированными. Работ, в которых бы 


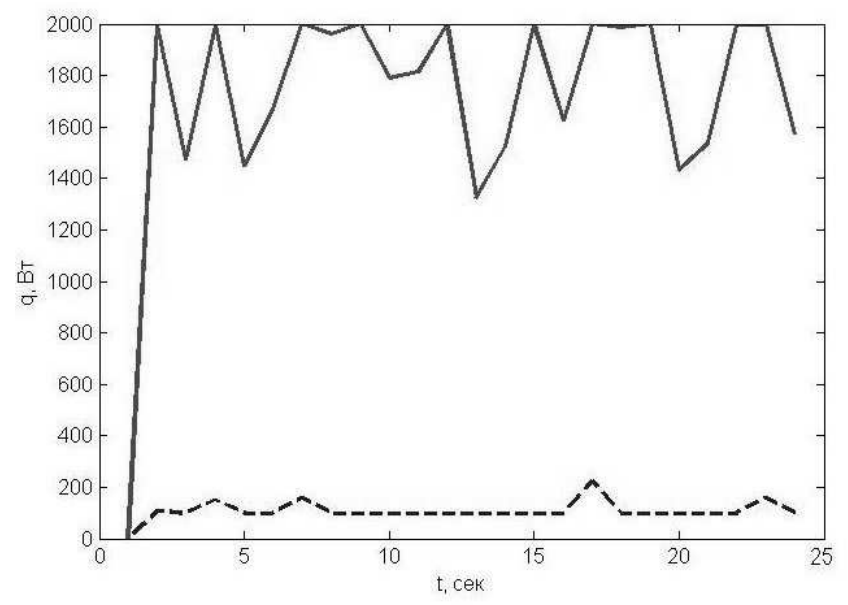

(a)

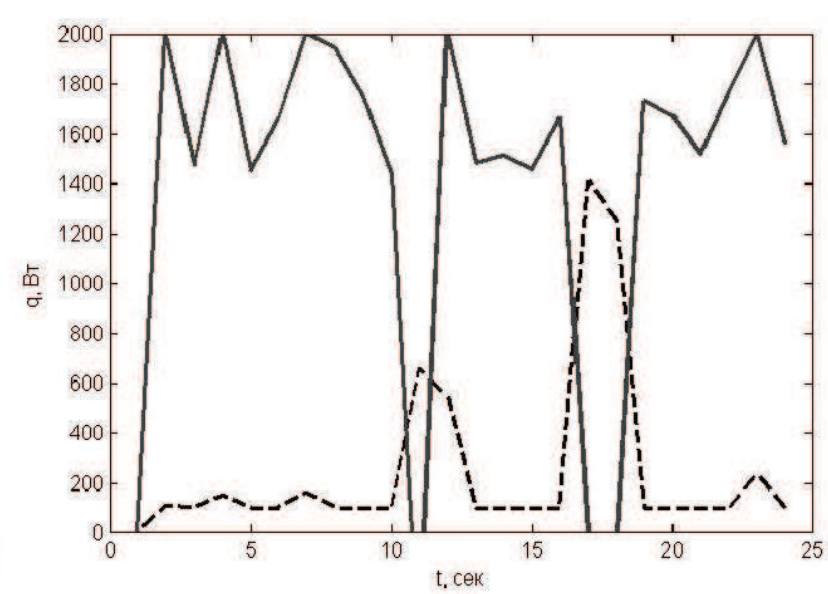

(b)

Рис. 4. Изменение расходов энергоносителей: (a) - при небольшом расходе $q_{i g}$; (b) - при возрастании расхода $q_{i g}$ в часы пик

решалась задача оптимального управления энергопотреблением и микроклиматом больших зданий с применением методов иерархической оптимизации на основе МРС-подхода при случайных ограничениях на ресурсы авторами, не найдено.

4. Заключение. Таким образом, авторами рассмотрен способ решения задачи оптимального управления сложной динамической системой со случайными ограничениями на ресурсы. Способ основан на использовании метода иерархической оптимизации, включающего итеративное решение локальных задач и задачи координации. Для решения локальных задач применяется МРСподход. Учет случайных ограничений на ресурсы приводит к решению одноэтапных задач стохастического программирования. Достоинством рассмотренного способа является то, что, используя простые линейные модели, он обеспечивает качественное оптимальное управление сложной системой при меняющихся ограничениях на доступные ресурсы. Результаты численного эксперимента подтвердили эффективность предложенного подхода.

\section{СПИСОК ЛИТЕРАТУРЫ}

1. Артамонов А. Г., Володин В. М., Авдеев В. Г. Математическое моделирование и оптимизация плазмохимических процессов. - М.: Химия, 1989.

2. Лэсдон Л. Оптимизация больших систем. - М.: Наука, 1975.

3. Мессарович М., Мако Д., Такахара И. Теория иерархических многоуровневых систем. - М.: Мир, 1973.

4. Сингх М., Титли А. Системы: декомпозиция, оптимизация и управление. - М.: Машиностроение, 1986.

5. Юдин Д. Б. Математические методы управления в условиях неполной информации: Задачи и методы стохастического программирования. - М.: КРАСАНД, 2017.

6. Farina M., Scattolini R. Distributed predictive control: A non-cooperative algorithm with neighbor-toneighbor communication for linear systems// Automatica. — 2012. — 48, № 6. - P. 1088-1096.

7. Findeisen W. Control and coordination in hierarchical system. - New York: Wiley, 1980.

8. Lamoudi M. Y., Alamir M. Beguery P. Distributed constrained model predictive control based on bundle method for building energy management// Proc. 50th IEEE Conf. on Decision and Control and European Control Conference (December 12-15, 2011, Orlando, FL, USA). — IEEE, 2011. — P. 8118-8124.

9. Mesbah A., Streif S., Findeisen R., Braatz R. D. Stochastic nonlinear model predictive control with probabilistic constraints// Am. Control Conf., 2014. - P. 2413-2419.

10. Moroşan P. D., Buisson J., Bourdais R. Distributed MPC for multi-zone temperature regulation with coupled constraints// IFAC Proc. Vol. — 2011. — 44, № 1. - P. 1552-1557. 
11. Moroşan P. D., Buisson J., Bourdais R. A distributed MPC strategy based on Benders decomposition applied to multi-source multizone temperature regulation// J. Process Control. — 2011. — 21. — P. 729737.

12. Scattolini R. Architectures for distributed and hierarchical Model Predictive Control. A review// J. Process Control. - 2009. - 19. - P. 723-731.

13. Wang L. Model Predictive Control System Design and Implementation using MATLAB. - London: Springer-Verlag, 2006.

Марьясин Олег Юрьевич

Ярославский государственный технический университет

E-mail: maryasin20030list.ru

Колодкина Анна Сергеевна

Ярославский промышленно-экономический колледж

E-mail: anuta_25@mail.ru 